OR, from 1978 to 1979 , he specified and developed a basic image processing library and a signal processing library addition for the array processor AP-120B. There he also analyzed $\mathrm{I} / \mathrm{O}$ times for the processor with attached disk. His current research and consulting at the University of Wyoming involves the array processor, and image, sonar and seismic signal processing.

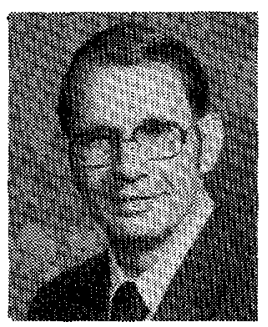

Dennis F. Moore (S'60-S'72-M'75) received the B.S. degree in electrical engineering from the University of Wyoming, Laramie, in 1965, the M.S. degree in electrical engineering from Colorado State University, Fort Collins, in 1971, and the $\mathrm{Ph} . \mathrm{D}$. degree from the University of Wyoming in 1975.

$\mathrm{He}$ is with the Laramie Energy Technology Center, a part of the Department of Energy, and has been employed by Collins Radio Company and Ampex Corporation. His duties at LETC include selection and development of data acquisition and control instrumentation, used in the development of in situ technology in the areas of coal gasification and tar sand and oil shale recovery methods. His research interests are in the areas of communications, signal processing and applications.

Dr. Moore is a member of Phi Kappa Phi, Sigma Xi, Tau Beta Pi, and is a Senior Member of the Instrument Society of America.

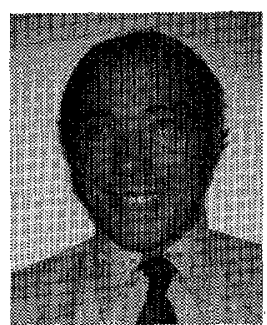

Robert F. Kubichek received the B.S. degrees in electrical engineering and computer science and the M.S. degree in electrical engineering in 1976 and 1977, respectively, all from the University of Wyoming, Laramie.

He was an engineer with Boeing Computer Services for two years, and is now back at the University of Wyoming, pursuing the Ph.D. degree in electrical engineering. He is currently a Research Assistant doing seismic signal processing work.

\title{
Time Delay Estimation in a Sensor Array
}

\author{
NORMAN L. OWSLEY, MEMBER, IEEE; AND GERALD R. SWOPE
}

\begin{abstract}
The estimation of time delays between elements in an array of $M$ point sensors located in a discrete multipath propagation medium is considered. It is shown that, in fact, time delays are not estimated directly. Rather, a location vector $\underline{\boldsymbol{\theta}}$ which parameterizes the relative source-receiving array geometry is estimated. In general, the relative sensor-to-sensor time delays are nonlinear functions of the location vector elements. The maximum-likelihood (ML) estimation process for $\boldsymbol{\theta}$ is established and shown to be realized by an extension of the socalled focused beam-former concept wherein wavefront shape matching time delays are generated. The form of the Cramér-Rao bound for the covariance matrix of the minimum mean-square error unbiased estimate of $\underline{\theta}$ is given.
\end{abstract}

\section{INTRODUCTION}

$\mathrm{T}$ $\mathrm{HE}$ problem of estimating the relative time delays between the outputs of sensors in a receiving array due to a single wavefront propagating to the array from a point source has been considered extensively within the context of estimating the range and bearing to the source [1]-[4]. In this previous work, the relative time delays can be expressed in terms of a vector $\boldsymbol{\theta}$ of unknown source location parameters, in conjunction with either the known or mean value geometry pa-

Manuscript received August 30,1980; revised December 4, 1980. This work summarizes research presented by the authors at the 1980 North Atlantic Treaty Organization (NATO) Advanced Study Institute (ASI) on Underwater Acoustics and Signal Processing (UASP), Copenhagen, Denmark. It also summarizes work completed since that time by these authors and others working in this field. Portions of this work were also presented at IEEE EASCON '79, Washington, DC, and the 1980 IEEE CDC, Albuquerque, NM.

The authors are with the Naval Underwater Systems Center, New London, CT 06320. rameters of the array. The assumption of spherical wavefront propagation is made. Then, the maximum-likelihood estimator array processing instrumentation for $\boldsymbol{\theta}$ is derived and its asymptotic performance is examined in terms of the Cramér-Rao variance lower bound. A dual class of problems could arise in certain types of sonar and navigation applications wherein the source location parameters are known but the geometry of the receiving array is to be established [5]. In this case, the vector $\underline{\boldsymbol{\theta}}$ to be estimated can represent an array geometry parameterization.

The previous work on time delay estimation has been typified by the assumption of a single wavefront, i.e., single path propagation from the source to the receiving array. This assumption can represent a considerable oversimplification of reality. In particular, the condition of multiple paths of source energy propagation to the receiving array more accurately characterizes the natural environment. To ignore this fact implies not only that a time delay measurement realization based on a single path model could exhibit degraded performance, but that potential performance gains which are available by an exploitation of the multipath condition are ignored [6]. Accordingly, in this paper we wish, first, to construct a single frequency model for the multipath medium which allows for both coherent and incoherent propagation along the constituent paths. Second, it is desired to establish the maximumlikelihood (ML) estimation process for the location vector $\boldsymbol{\theta}$ when multipath is present. Finally, the approach to evaluating the asymptotic performance of the ML estimator is stated in terms of the Cramer-Rao bound with particular attention given to the effect of multipath. 


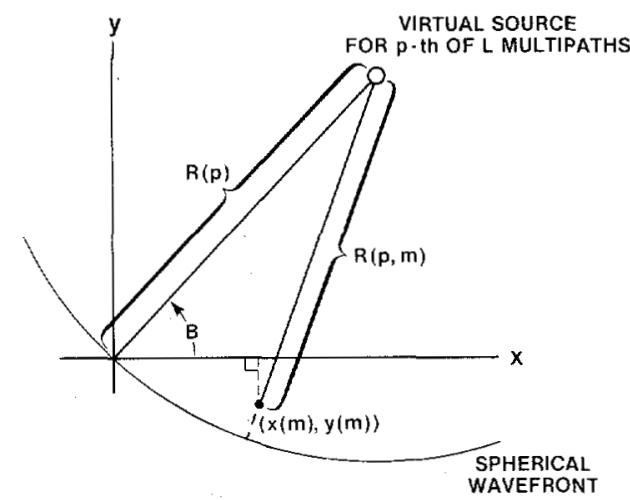

Fig. 1. Illustration of the relative effective horizontal plane geometry for propagation from a source to the $m$ th sensor in receiving array along the $p$ th of $L$ paths.

\section{A Multipath Model}

Consider the $p$ th of $L$ spherical wavefronts arriving at the $m$ th sensor of an array consisting of $M$ point sensors as illustrated in Fig. 1. The distance $R(p)$ is the distance measured along the $p$ th multipath from the source to the origin of a Cartesian coordinate system centered at the array. The distance $R(p)$ is the radius of curvature for the $p$ th spherical wavefront and can be envisioned as an effective horizontal range to a virtual source. The distance $R(p, m)$ is the corresponding distance along the $p$ th multipath from the source to the $m$ th sensor in the array having coordinates $(x(m), y(m))$ in the horizontal plane. For example, for the source location problem, the location vector $\boldsymbol{\theta}$, defined by its transpose as

$$
\underline{\boldsymbol{Q}}^{T}=[B, R, d]
$$

has the elements bearing $B$, horizontal range $R$, and source depth $d$. These location parameters are used in conjunction with a propagation model for the medium to obtain the path radii $R(p), p=1,2, \cdots, L$. Because the array shape in the horizontal plane can be expressed in terms of the sensor coordinates $(x(m), y(m)), m=1,2, \cdots, M$, all required estimated path lengths

$$
R(p, m)=\left[R^{2}(p) \sin ^{2} B+h^{2}(p, m)+2 R(p) y(m) \sin B\right]^{1 / 2}
$$

with

$$
h(p, m)=\left[(R(p) \cos B-x(m))^{2}+y^{2}(m)\right]^{1 / 2}
$$

from the source to the $m$ th sensor along the $p$ th path can be formulated. The effective horizontal range $R(p)$ along the $p$ th path is directly determined from the actual horizontal range $R$ and the source depth $d$ by a strictly deterministic consideration of the relative source-array geometry. It follows that the propagation time along the $p$ th path to the $m$ th sensor can be expressed as

$$
D(p, m)=R(p, m) / c
$$

where $c$ is an average value of energy propagation velocity along the $p$ th path. In actual practice it could perhaps be necessary to use the expression

$$
D(p, m)=\sum_{i=1}^{K} \frac{R_{i}(p, m)}{c_{i}(p)}
$$

in lieu of (4) [8]. In this form, a different propagation velocity $c_{i}(p)$ can be accommodated over the $i$ th of $K$ segments which comprise the total source-to-element propagation path of length $R(p, m)$ given by

$$
R(p, m)=\sum_{i=1}^{K} R_{i}(p, m) .
$$

It should be evident that the definition of signal propagation time and propagation modeling issues cannot be separated in the discrete multipath time delay estimation problem.

Let the signal and noise components at a sensor be zeromean, stationary, and uncorrelated Gaussian random processes. Specifically, consider the output of the $m$ th sensor to be given by the time domain expression

$$
r_{m}(t)=\sum_{p=1}^{L} a(p) s_{p}(t+D(p, m))+v_{m}(t)
$$

where the $L$ multipath signals $s_{p}(t)$ are assumed to be zeromean Gaussian random processes. The term $a(p)$ is the relative attenuation factor of the signal due to assumed ideal spherical spreading on the $p$ th of $L$ multipaths. These attenuation factors are defined so that

$$
\sum_{p=1}^{L} a^{2}(p)=1
$$

where $a(p)$ is obtained by a propagation model path length consideration. Equation (7) implies that the wavefront arriving at the array on a single propagation path is perfectly coherent. That is, the sensor outputs due to the signal on a particular path differ only by the propagation time $D(p, m)$ which can be expressed directly in terms of the source and array geometry parameters.

Given the space-time signal plus noise model above, it is desired to express this received waveform in the discrete frequency domain for the purpose of separating pure time delay and path-to-path correlation effects. With this objective, let the $m$ th sensor output of (7) be operated on by the sequence of $K$ discrete Fourier transforms at frequency $f$ represented by

$$
\begin{array}{r}
r_{m}(f, k)=\int_{(k-1) T}^{T} r_{m}(t) \exp (-j 2 \pi f t) d t \\
\\
\quad(k=1,2, \cdots, K) .
\end{array}
$$

Formally, it is assumed that the discrete Fourier transform interval $T$ is large compared to the maximum path propagation time, i.e., $T \gg \max [D(p, m)]$. However, in actual practice it is adequate to require that $T \gg \max [|D(p, m)-D(q, n)|]$ because it is only these time differences which must necessarily enter into the location vector $\boldsymbol{\theta}$ maximum-likelihood estimator realization obtained in the following section. Using (7) in (9) gives the discrete Fourier coefficient at time $k T$

$$
r_{m}(f, k)=\sum_{p=1}^{L} a(p) s(k, p) \exp (-j 2 \pi f D(p, m))+v_{m}(k)
$$

with the $p$ th multipath signal component 


$$
s(k, p)=\int_{(k-1) T}^{k T} s_{p}(t) \exp (-j 2 \pi f t) d t .
$$

Let the signal discrete Fourier coefficient of (11) have covariance

$$
E\left\{s^{*}(k, p) s(r, q)\right\}=\frac{1}{L} \sigma_{s}^{2} \rho(p, q) \delta_{k r}
$$

where $\sigma_{s}^{2}$ is the total expected signal power at a single sensor in the array due to energy arriving on all propagation paths and contained in the discrete Fourier coefficient of $(9), \rho(p, q)$ is the complex coherence between the $p$ th and $q$ th path signals, and $\delta_{k r}$ is the discrete Dirac delta function. Similarly, the additive noise term $v_{m}(k)$ can be shown to be a complex, zero-mean, stationary Gaussian independent discrete random sequence with covariance

$$
E\left\{v_{m}(k) v_{n}^{*}(r)\right\}=\sigma^{2} q(m, n) \delta_{k r} .
$$

With the above definitions, it is convenient to introduce the vector notation

$$
\underline{\boldsymbol{R}}(k)^{T}=\left[r_{1}(f, k) r_{2}(f, k) \cdots r_{M}(f, k)\right]
$$

for the transpose of a complex $M$-vector consisting of the discrete Fourier coefficients for the $M$ sensor outputs corresponding to the interval $(k-1) T, k T$. Now an $M$-by- $M$ cross-spectral density matrix for the array at frequency $f$ can be defined as

$$
\begin{aligned}
\boldsymbol{P} & =E\left\{\underline{\boldsymbol{R}}(k) \underline{\boldsymbol{R}}(l)^{*}\right\} \delta_{k l} \\
& =\sigma_{s}^{2} \boldsymbol{D} E D^{*}+\sigma^{2} \boldsymbol{Q}
\end{aligned}
$$

where $\operatorname{Tr}\{Q\}=M$. The notation "*" indicates the matrix complex conjugate transpose operation. In (15), $\boldsymbol{D}$ is an $\boldsymbol{M}$-by- $\boldsymbol{L}$ delay vector matrix with the $p$ th column having the $m$ th element $\exp (-j 2 \pi f D(p, m)), \boldsymbol{E}$ is an $L$-by- $L$ path correlation matrix with $p q$ th element $a(p) a(q) \rho(p, q)$, and $Q$ is the noiseonly normalized cross-spectral density matrix at frequency $f$ with the $m n$th element given by $q(m, n)$.

Having provided a statistical model describing the multipath signal and additive noise environment for the $M$-element sensor array, it is noted that only the delay vector matrix $D$ is a strong function of the location vector $\boldsymbol{\theta}$ which is to be estimated. The next section assumes that the received data $K M$ vector $\underline{R}$ with

$$
\underline{R}^{T}=\left[\underline{R}(1)^{T} \underline{R}(2)^{T} \cdots \underline{R}(K)^{T}\right]
$$

is available and subjects these data to a maximum-likelihood estimator development with respect to the assumed deterministic, but unknown, location vector $\boldsymbol{\theta}$.

\section{The Maximum-Likelihood Estimator}

Given the composite received data vector of discrete Fourier coefficient vectors $\underline{R}$ in (16), the log-likelihood function

$$
\log (p(\underline{R} / \underline{\boldsymbol{\theta}}))=-K \log |\boldsymbol{P}|-\sum_{k=1}^{K} \underline{\boldsymbol{R}}(k)^{*} \boldsymbol{P}^{-1} \underline{\boldsymbol{R}}(k)
$$

can be written. In (17), $p(\underline{R} / \mathbf{\theta})$ is the complex multivariate Gaussian conditional probability density function of $\underline{R}$, given the location vector $\boldsymbol{\theta}$, and $|\boldsymbol{P}|$ is the determinant of the array cross-spectral density matrix $\boldsymbol{P}$. Using the identity

$$
\boldsymbol{P}^{-1}=\frac{1}{\sigma^{2}}\left[Q^{-1}-Q^{-1} D\left[D^{*} Q^{-1} D+\frac{\sigma^{2}}{\sigma_{s}^{2}} E^{-1}\right]^{-1} D^{*} Q^{-1}\right]
$$

and the definition

$$
\hat{\boldsymbol{P}}=\frac{1}{K} \sum_{k=1}^{K} \underline{R}(k) \underline{R}(k)^{*}
$$

as an estimate of the array cross-spectral density matrix allows (17) to be written

$$
\begin{aligned}
\log (p(\underline{\boldsymbol{R}} / \underline{\mathbf{Q}}))= & K \log |\boldsymbol{P}|-\frac{1}{\sigma^{2}} \sum_{k=1}^{K} \underline{\boldsymbol{R}}(k)^{*} Q^{-1} \underline{\boldsymbol{R}}(k) \\
& +\frac{K}{\sigma^{2}} \sum_{p=1}^{L} \sum_{q=1}^{L} v(p, q) \underline{D}(q)^{*} \boldsymbol{Q}^{-1} \hat{\boldsymbol{P}} Q^{-1} \underline{D}(p) .
\end{aligned}
$$

In (20), $v(p, q)$ is the $p q$ th element in the $L$-by $L$ matrix $\left[D^{*} Q^{-1} D+\left(\sigma^{2} / \sigma_{s}^{2}\right) E^{-1}\right]^{-1}$ and $D(p)$ is the $p$ th column in the delay vector matrix $D$. The second term on the right-hand side of (20) is not a function of the location vector $\boldsymbol{\theta}$ with respect to which the log-likelihood function is to be maximized. Therefore, by dropping the second term and introducing the notation

$$
y(p, k)=\underline{D}(p)^{*} Q^{-1} \underline{R}(k),
$$

(20) can be rewritten as

$$
\begin{aligned}
L(\underline{R} / \underline{\Theta})= & -K \log |\boldsymbol{P}|+ \\
& +\frac{1}{\sigma^{2}} \sum_{p=1}^{L} \sum_{q=1}^{L} v(p, q) \sum_{k=1}^{K} y(q, k) y(p, k)^{*} .
\end{aligned}
$$

The term $y(p, k)$ defined by $(21)$ is referred to as the focused beam-former output for the $p$ th of $L$ multipath signal arrivals. The direct realization of the likelihood function as given in (22) is illustrated in Fig. 2. The components of a system to evaluate the $\log$-likelihood $L(\underline{\boldsymbol{R}} / \underline{\boldsymbol{\theta}})$ for a given estimate of $\underline{\boldsymbol{\theta}}$, namely, $\hat{\boldsymbol{\Theta}}$, are

1) a spatial noise prewhitening matrix filter $Q^{-1}$;

2) a bank of $L$ focused beam-formers $\underline{D}(p)(p=1,1, \cdots, L)$ matched to the time delays of each of the supposed spherical wavefronts defined in terms of the location (geometry) vector estimate $\hat{\boldsymbol{\Theta}}$ for either the source or the array;

3) a matrix correlator which cross correlates the pairwise focused beam-former outputs from the $L$ possible outputs using a $K$ point time average; and

4) a coherent (complex) linear combination of the crosscorrelated focused beam-former outputs weighted by the term $v(p, q)$ with a final removal of the bias term $K \log |\boldsymbol{P}|$.

It is observed that the focused beam-former delay vectors $\underline{D}(p)(p=1,2, \cdots, L)$, the combiner coefficients

$$
v(p, q)=\left[\left[D^{*} Q^{-1} D+\frac{\sigma^{2}}{\sigma_{s}^{2}} E^{-1}\right]^{-1}\right]_{p, q}
$$

and the bias removal term $K \log |\boldsymbol{P}|$ are all functions of the 


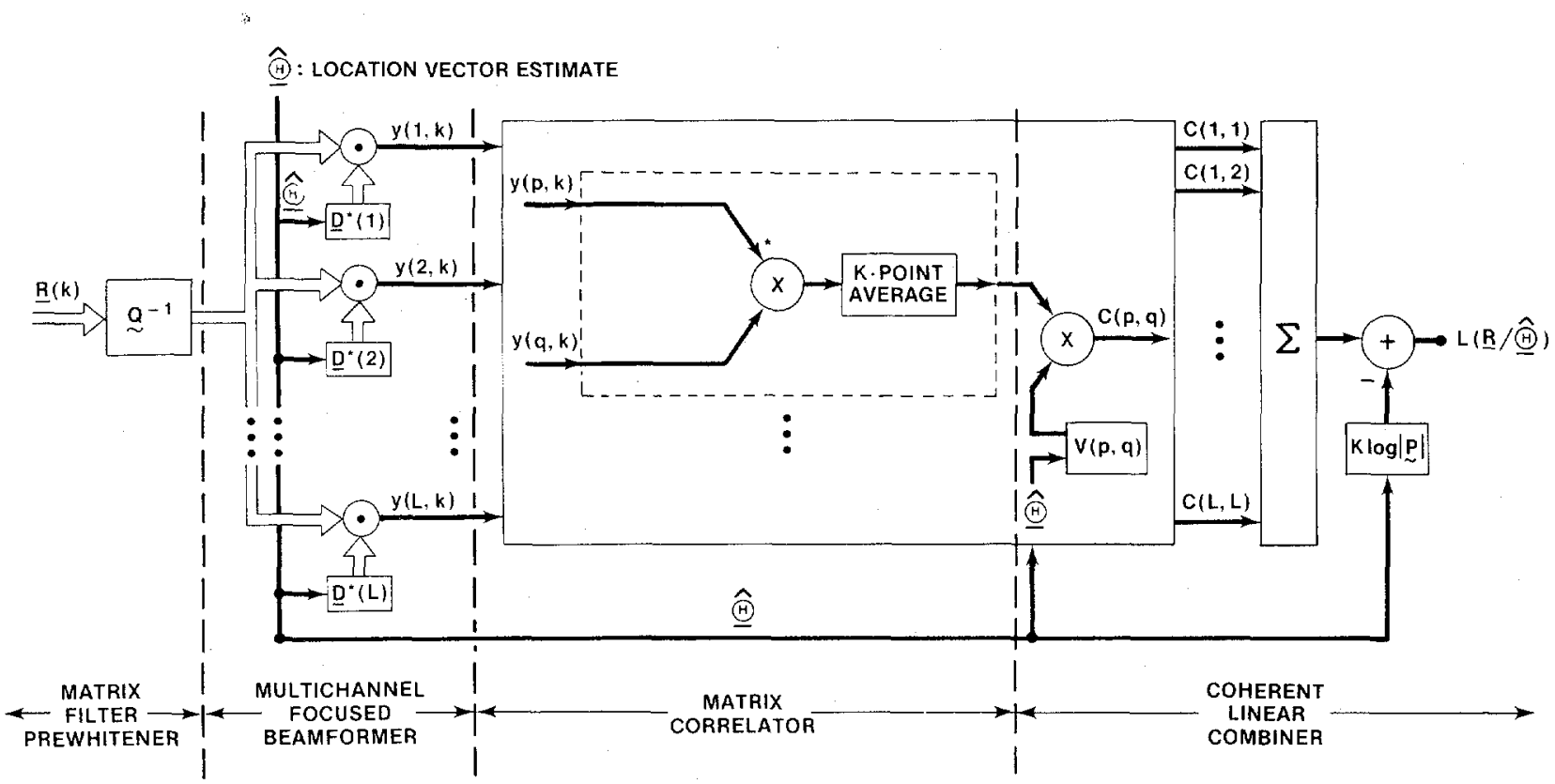

Fig. 2. A direct realization of the maximum-likelihood estimation process for the location vector $\boldsymbol{\theta}$ given $L$ discrete source-to-receiver propagation paths.

estimate $\hat{\boldsymbol{\theta}}$. For the low signal-to-noise ratio (SNR) case $\left(M \sigma_{s}^{2} / \sigma^{2}\right)<<1$, it follows from (23) that

$$
v(p, q)=\left(\sigma_{s}^{2} / \sigma^{2}\right) a(p) a(q) \rho(p, q) .
$$

Thus, if the various multipaths are incoherent with $\delta(p, q)=$ $\rho_{p q}$, then the estimated focused beam-former power outputs are simply weighted by the path SNR, $\left(\sigma_{s}^{2} / \sigma^{2}\right) a^{2}(p), p=$ $1,2, \cdots, L$ and accumulated. Otherwise, the focused beamformer output cross correlations are weighted by a term $a(p)$. $a(q) \rho(p, q)$ proportional to the correlation before combining. For the high SNR case, it can be seen from (23) that path-topath correlation terms in the $E$-matrix inverse are dominated by quantities involving the generalized cosine $\underline{D}(p)^{*} Q^{-1} \underline{D}(q) / M$ between the $p$ th and $q$ th wavefront delay vectors. For example, consider the case of spatially uncorrelated and uniform background noise where $Q=I$ an $M$-by $M$ indentity matrix. Now, if the delay vectors are orthogonal with $\underline{D} *(p) \underline{D}(q) / M=$ $\delta_{p q}$, then (22) requires that the estimated focused beam-former output power be combined with uniform weighting.

For the spatially uncorrelated background noise case, the maximization of (22) with respect to $\boldsymbol{\Theta}$ can be efficiently implemented by a numerical search procedure based on an orthogonal decomposition of the estimated cross-spectral density matrix of (19) [6]. Otherwise, the direct implementation of Fig. 2 can be realized with some degree of efficiency deriving from the complex conjugate symmetry of the linear combiner inputs: Whether or not $L(\boldsymbol{R} / \boldsymbol{\theta})$ has a unique global maximum with respect to $\boldsymbol{\theta}$ is strictly a function of the sensor locations in the array. Generally speaking, the higher the percentage of sensors which are located at distances on the order of one-half a wavelength $(\lambda / 2)=(f / 2 c)$ at the focusing frequency $f$, the more distinguishable the global maximum will be from the local ambiguous maxima. Conversely, widely spaced sensors would lead to significant estimator ambiguity unless some form of a priori knowledge on the parameters to be estimated can be exploited.

\section{Estimator Performance}

If an unbiased estimate of the location vector $\underline{\boldsymbol{\theta}}$ is required and mean-square error (MSE) is used as a measure of estimator performance, then an absolute bound on this performance can be obtained. In particular, the covariance matrix of the minimum MSE unbiased estimate $\underline{\hat{\boldsymbol{\theta}}}$ of $\underline{\boldsymbol{\theta}}$ is given by

$$
\operatorname{cov}(\underline{\hat{\boldsymbol{\Theta}}})=\boldsymbol{J}^{-1}(\underline{\boldsymbol{\theta}})
$$

where $\boldsymbol{J}(\boldsymbol{\Theta})$ is known as the Fisher information matrix. The matrix $\boldsymbol{J}(\underline{\boldsymbol{Q}})$ has $i j$ th term given by

$$
[\boldsymbol{J}(\underline{\boldsymbol{\theta}})]_{i j}=-E\left\{\frac{\partial^{2} L(\underline{\boldsymbol{R}} / \boldsymbol{\theta})}{\partial \theta_{i} \partial \theta_{j}}\right\}
$$

where $\Theta_{i}$ is the $i$ th element of the location vector $\boldsymbol{\Theta}$. Equation (24) is the vector form of the Cramér-Rao lower bound. The maximum-likelihood estimate of $\boldsymbol{\theta}$ for long observation time, i.e., large $K$, is known to exhibit a covariance matrix which approaches (24). Thus, a preliminary understanding of this bound is relevant to obtaining a statement of performance for the ML procedure defined in the previous section.

Because the statistics of the signal and noise components of the received data $\boldsymbol{R}(k)$ are zero mean and Gaussian with covariance matrix $\boldsymbol{P}=\boldsymbol{P}(\underline{\boldsymbol{\theta}})$ given by (15), (25) can be written as

$$
\begin{aligned}
& {[\boldsymbol{J}(\boldsymbol{\Theta})]_{i j} } \\
&=\operatorname{Tr}\left\{\boldsymbol{P}^{-1} \frac{\partial \boldsymbol{P}}{\partial \Theta_{i}} \boldsymbol{P}^{-1} \frac{\partial \boldsymbol{P}}{\partial \Theta_{j}}\right\} \quad[7] \\
&=4 \sigma_{s}^{4} K \operatorname{Tr}\left\{\boldsymbol{P}^{-1} \sum_{p=1}^{L} \sum_{m=1}^{m} \operatorname{Re}\left\{D \boldsymbol{E} \frac{\partial D^{*}}{\partial D(p, m)}\right\}\right. \\
&\left.. \frac{\partial D(p, m)}{\partial \Theta_{i}} \boldsymbol{P}^{-1} \sum_{p=1}^{L} \sum_{m=1}^{M} \operatorname{Re}\left\{\boldsymbol{D E} \frac{\partial \boldsymbol{D}}{\partial D(p, m)}\right\} \frac{\partial D(p, m)}{\partial \Theta_{j}}\right\}
\end{aligned}
$$


Evaluation of (26) in its general form, at the very least, is a formidable task and is not attempted herein. However, because the path-to-path correlation matrix $\boldsymbol{E}$ and the cross-spectral density matrix $\boldsymbol{P}$ are both positive definite, an increase in the number of multipaths $L$ results in a corresponding increase in the magnitude of the Fisher matrix term $[\boldsymbol{J}(\underline{\boldsymbol{\theta}})]_{i j}$. It follows that the availability of multipath structure information contributes to an associated overall decrease in the terms of the estimator variance matrix lower bound.

Finally, evaluation of the partial derivatives $\partial D(p, m) / \partial \Theta_{i}$ in (26) for all $p, m$, and $i$ is strictly a function of the problem and its particular geometry, i.e., either source location or array shape estimation. Furthermore, this evaluation is dependent on the geometric model parameterization inclusive of coordinate system origin and any geometric model approximations which are invoked. These considerations are beyond the intent of this paper which is limited to the presentation of a general framework for time delay related parameter estimation and related performance evaluation using a sensor array receiving a single frequency signal from a source in a multipath propagation environment.

\section{Conclusions}

The implementation structure for maximum-likelihood estimation of time delay related geometric parameters in the presence of multipath propagation to a sensor array from a single frequency source is invariant with respect to the specific type of problem. That is, whether the problem at hand is the estimation of wavefront shape, as in source location, or receiving array shape and orientation estimation, the relative wavefront/ array shape geometry can be parameterized and the same focused beam-former structure can be used for the geometric parameter estimation. The use of multipath propagation structure information can serve to improve performance of the estimation process for geometry-dependent time delays. However, it is the need to incorporate a multipath prediction model into the estimation processor design which constitutes a significant implication of this work. Accordingly, future work should concentrate on the determination of sensitivity of estimator performance to either errors in or total lack of such a deterministic multipath propagation model.

\section{ACKNOWLEDGMENT}

The authors wish to thank Prof. P. M. Schultheiss, Yale University, for his interest in and useful discussions regarding the work reported herein.

\section{REFERENCES}

[1] W. J. Bangs and P. M. Schultheiss, "Space-time processing for optimal parameter estimation," in Signal Processing, J. W. R. Griffiths, P. L. Stocklin, and C. van Schooneveld, Eds. New York: Academic, 1973 , pp. 577-590.

[2] W. R. Hahn, "Optimum signal processing for passive sonar range and bearing estimation," J. Acoust. Soc. Amer., vol. 58, pp. 201207,1975 .

[3] G. C. Carter, "Variance bounds for passively locating an acoustic source with a symmetric line array," $J$. Acoust. Soc. Amer., vol. 62, pp. 922-926, 1977.

[4] P. M. Schultheiss and J. P. Ianniello, "Optimum range and bearing estimation with randomly perturbed arrays,"J. Acoust. Soc. Amer., vol. 68 , pp. $167-173,1980$.

[5] N. L. Owsley and G. R. Swope, "Array shape determination using time delay estimation procedures," in Proc. IEEE EASCON ' 80 , Sept. 1980, pp. 158-165.

[6] N. L. Owsley, "Source location with an adaptively focused array," presented at the IEEE Conf. Decision and Contr., Dec. 10-12, 1980.

[7] W. J. Bangs, "Array processing with generalized beamformers," Ph.D. dissertation, Dep. Eng. Appl. Sci., Yale Univ., New Haven, CT, 1971 ,

[8] R. C. Spindel, "Multipath processing for ocean acoustic tomography," in Proc. IEEE EASCON '80, Sept. 1980, pp. 165-170.

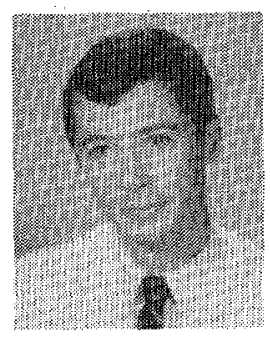

Norman L. Owsley (S'63-M'68) was born in Cleveland, $\mathrm{OH}$, on June 24,1941 . He received the B.S.E.E. degree from Lehigh University, Bethlehem, PA, in 1963, and the M.S. and Ph.D. degrees from Duke University, Durham, NC, in 1965 and 1968 , respectively.

Since 1968 he has been with the Naval Underwater Systems Center, New London, CT. In addition, he has served as an Instructor at the University of Connecticut, Storrs, and the University of New Haven, New Haven, CT, as well as the Rensselaer Polytechnic Institute, Troy, NY, teaching courses in detection and estimation theory. In 1977 he served as a NATO Exchange Scientist to Norway. While at NUSC, he has conducted research in nearly all aspects of digital signal processing related to underwater acoustics. His current interests are adaptive techniques for signal parameter estimation and acoustic source location.

Dr. Owsley is a member of Eta Kappa Nu, Pi Mu Epsilon, and Sigma $\mathrm{Xi}$.

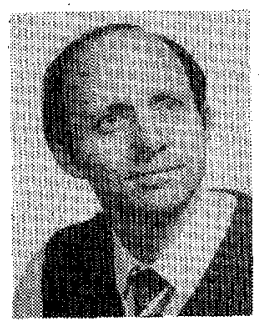

Gerald R. Swope received the B.S. degree in mathematics from Millersville State College, Millersville, PA, in 1965, and the M.S. degree in mathematics from Lehigh University, Bethlehem, PA, in 1967.

Since 1967 he has been with the Naval Underwater Systems Center, New London, CT. From 1970 to 1975 he served as a Mathematician at NUSC's Bermuda Research Detachment. It was on this assignment that he became interested in digital signal processing. His current interests are multivariate stochastic processes and optimization. He is also pursuing the Ph.D. degree in operations research and statistics at Rensselaer Polytechnic Institute, Troy, NY. 